\title{
La productividad en operaciones de encofrado. Caso de estudio: Edificio de Ciencias de la Salud UNAH-VS
}

Coralia Edith Zelaya, Fanny Marisela Ortiz Flores, Gerardo José Barralaga, Josué Molina Medina

Guillermo A. Recarte Suazo²

\section{RESUMEN}

El estudio se realizó en el Edificio de Ciencias de la Salud UNAH-VS, con el objetivo de describir cómo es la productividad en operaciones de encofrado utilizando la técnica del análisis de actividad; además, para determinar cómo distribuye su tiempo una cuadrilla en una operación de encofrado, cómo varía esta distribución del tiempo durante el proceso productivo, analizar al aporte de cada uno de los miembros de la cuadrilla y, finalmente, entender las ventajas de aplicar la técnica del análisis de actividad sobre el muestreo del trabajo.

El análisis de actividad es una técnica basada en procesos cuantitativos, no experimental, que se utilizó de manera transversal para describir la productividad, haciendo uso de siete categorías: trabajo directo, preparatorio, manejo de materiales, manejo de equipo y herramientas, esperas, viajes y personal. La población de estudio seleccionada fueron las cuadrillas trabajando en los encofrados de este proyecto. Se utilizó el método de muestreo de la cuadrilla, enfocándose en una de ellas. Se hicieron un mínimo de 45 observaciones al azar por hora, teniendo en consideración un error permisible del $5 \%$, un nivel de confianza del $95 \%$ y una proporción de ocurrencias del $50 \%$.

El trabajo directo que la cuadrilla dedicó al proceso fue de $31.82 \%$; esperas, $35.45 \%$ y personal, $16.36 \%$. En el transcurso del proceso se pudo observar cómo disminuía la productividad al acercarse el mediodía y el grado de esfuerzo que cada miembro de la cuadrilla aportó al producto. Los resultados descriptivos sirven de indicadores de problemas y sus posibles causas y soluciones.

Palabras clave: análisis de actividad, productividad, muestreo, operaciones de construcción, encofrado.

\footnotetext{
${ }^{1}$ Estudiantes de la Maestría en Ingeniería de la Construcción y Gerencia de Proyectos, UNAH-VS.

${ }^{2}$ Asesor, Maestría en Ingeniería de la Construcción y Gerencia de Proyectos, coordinador Depto. de Ingeniería Civil,UNAH-VS, dirección electrónica: maestria.ing.const.unah.vs@gmail.com
} 


\section{ABSTRACT}

The study was conducted in the Building Health Sciences UNAH-VS. The overall objective is to describe how productivity formwork operations and has specific objectives, determine how you distribute your time a gang in an operation of formwork, as this distribution varies time during the production process, to analyze the contribution of each of gang members, and finally understand the advantages of applying the technique of activity analysis of work sampling .

Activity analysis is a technique based on quantitative processes, not experimental, so cross was used to describe productivity using seven categories: direct labor, preparatory material handling equipment and tools management, delays, travel and staff. The study population was selected crews working on the forms for this project. We used the sampling method of the gang, focusing on one of them, there were a minimum of 45 random observations per hour, taking into consideration allowable error of $5 \%$, a confidence level of $95 \%$ and a ratio of occurrences $50 \%$.

Direct work the crew dedicated to the process was $31.82 \%$; expect, $35.45 \%$, and staff, $16.36 \%$. In the course of the process was observed as decreased productivity approaching noon. It was observed the level of effort that each member of the band contributed to the product. The descriptive results are indicators of problems and their possible causes, and solutions.

Keywords: activity analysis, productivity, sampling, construction operations, formwork.

\section{INTRODUCCIÓN}

En enero de 2013, a un grupo de estudiantes de la Maestría de Ingeniería de la Construcción y Gerencia de Proyectos (MICGP), en la clase de Planeamiento y Mejoramiento de Métodos de la Construcción, como parte del trabajo asignado, se les pidió que hicieron mediciones de la productividad presente en diferentes obras de construcción que se desarrollaban en la ciudad de San Pedro Sula.

Uno de estos grupos centró su trabajo en operaciones de encofrado de columnas de concreto en el segundo nivel del edificio principal del complejo de la Escuela de Ciencias de la Salud de la UNAH-VS. Para tales efectos, se usaron videos que ilustran la operación y se propuso describir la productividad de dichas operaciones haciendo uso de la técnica del análisis de actividades, que es un nuevo método desarrollado por el Instituto de la Industria de la Construcción como una extensión del muestreo del trabajo. 
En Estados Unidos, el interés por el estudio de la productividad en la industria de la construcción no es un tema nuevo, así, en la década de los 70, reconociendo el aporte económico que la industria de la construcción hace a sus respectivos países, la Mesa Redonda de Negocios inicia a estudiarla. En Chile, los autores (Ramírez, Alarcón y Knights, 2004) y Alarcón sostienen que la globalización y la nuevas necesidades de los clientes ha aumentado la competencia, obligando a las constructoras a actualizarse para alcanzar niveles de competitividad.

En Honduras, la industria de la construcción sufre una situación similar a la citada anteriormente en Chile, instituciones nacionales como COALIANZA e internacionales como Banco Mundial (Proceso Digital, 2012; Diario La Prensa, 2011) han limitado la participación de las empresas constructoras nacionales en la ejecución de importantes proyectos de construcción, pero, poco o ningún esfuerzo se ha dedicado al estudio del tema, a pesar del efecto adicional que ha tenido la crisis política y financiera que sufre el país.

En este contexto, se estableció como objetivo general del estudio describir cómo es la productividad en las operaciones de encofrado en el Edificio de Ciencias de la Salud UNAH-VS. Para esto se establecieron como objetivos específicos: determinar cómo distribuye su tiempo una cuadrilla en una operación de encofrado, establecer como varía esta distribución del tiempo dedicado por la cuadrilla hora a hora, analizar cómo distribuyen su tiempo cada uno de los miembros de la cuadrilla en este período durante la misma operación y, finalmente, entender las ventajas de aplicar la técnica del análisis de actividad sobre el muestreo del trabajo.

El estudio y mejora de la productividad en la construcción tiene un resultado directo en las empresas constructoras, porque, como bien lo señalaba la Mesa Redonda de Negocios, este resultado se convierte directa o indirectamente en mayor circulante, mayor contribución al PIB, más fuentes de trabajo; en resumen, más dinero para todos, de allí la importancia práctica y social del estudio. Además, se genera conocimiento sobre la productividad en este tipo de operaciones en nuestro país.

\section{ANTECEDENTES}

Oglesby, Parker y Howell definen la productividad como una dimensión del desempeño, enfocándose en el desempeño del proyecto: "Un esfuerzo por medir la efectividad con la que las capacidades de gestión, trabajadores, materiales, equipo, herramientas y espacio de trabajo son empleados en apoyo a las actividades de cara al trabajo para producir un edificio terminado, planta, estructura u otra instalación fija al más bajo costo factible". Por su parte, Serpell la define como: "Una medición de la 
eficiencia con que los recursos son administrados para completar un producto específico, dentro de un plazo establecido y con un estándar de calidad dado".

Siguiendo a Oglesby, Parker y Howel, hay cuatro partes que juegan un rol en un proyecto de construcción: el dueño, el equipo de diseño, el equipo de constructores y la fuerza laboral. Aunque el control de los recursos necesarios para trabajar de manera eficaz y eficientemente está en manos de los tres primeros, terminar dentro de presupuesto con la calidad especificada depende en gran parte de las habilidades de la fuerza laboral y de su deseo de realizar el trabajo. La otra parte depende de que las tres primeras desempeñen adecuadamente su rol.

Varios autores (Martínez, Verbal y Serpell, 1990; Serpell, 2002) hacen hincapié en cinco contramedidas para contrarrestar los factores que disminuyen la productividad en obras de construcción. La primera de ellas está relacionada con aspectos motivacionales de la fuerza laboral; la segunda, enfatiza la capacitación de artesanos, capataces y profesionales de la construcción; la tercera, involucra la implementación de procesos de planificación de corto plazo orientado a las cuadrillas y sistemas de control de materiales, recurso humano, costo y tiempo; finalmente, la planificación detallada de operaciones de construcción, tales como planteles del proyecto y la metodología de los trabajos.

También ha sido comprobado por diferentes autores, que con la implementación de programas de mejoramiento de la productividad se deberían obtener incrementos de entre el 20 y 40 \% (Martínez y Alarcón, 1988). Al respecto, un caso de estudio realizado en construcciones mecánicas y eléctricas en Inglaterra, logró incrementos de productividad en un $116 \%$, comparado con $37 \%$ del método tradicional, utilizando un sistema "lean" y ágil, reduciendo riesgos de salud, seguridad y sin retrasos ni compresiones (Court, Pasquire y Gibb, 2009).

Martínez y Alarcón propone un programa de estudio de la productividad en cuatro etapas: la primera, es la etapa de diagnóstico basado en la utilización de las siguientes herramientas: observación directa, muestreos del trabajo, análisis de la información de costos, análisis del programa y puntos de control, información de rendimientos, encuestas de detención y cuestionarios a los obreros. La segunda etapa consiste en el desarrollo de un programa de mejoramiento de la productividad; la siguiente etapa es el la implementación del programa y, finalmente, el seguimiento y control aplicando una serie de técnicas: encuestas de detención de los capataces, muestreos del trabajo y cuestionarios a los obreros. En el presente trabajo nos enfocaremos en la etapa de diagnóstico del proceso. 
En el desarrollo de un proyecto de construcción, según Oglesby, Parker y Howel, hay cuatro partes involucradas: el dueño, el equipo de diseño, el equipo de constructores y la fuerza laboral. El realizar los trabajos asignados depende en gran manera de las habilidades de la fuerza laboral y de su deseo de realizar el trabajo, razón por la cual medir el desempeño de la fuerza laboral se convierte en vital para una buena gestión de proyectos de construcción.

Asimismo, diversos autores (Oglesby, Parker y Howell, 1989; Serpell, 2002; Jenkins y Orth, 2003; Construction Industry Institute, 2010; Alarcón, 2012) han señalado diferentes formas de medir el desempeño de la fuerza laboral a través de tasas unitarias directas de la productividad, tales como la productividad de factores, la productividad laboral y la productividad del desempeño; pero también se puede estimar por medio de la cantidad de trabajo que tiene que ser rehecho. Finalmente, existen varias técnicas basadas en la observación y muestreo del trabajo, descritos en la literatura, entre los que se incluyen: muestreos de campo, muestreos de productividad, muestreos de 5 minutos y el análisis de actividades.

\section{METODOLOGÍA}

El análisis de actividad puede describirse como una técnica basada en procesos cuantitativos no experimental, porque está basada en el muestreo del trabajo que el Cll define como una herramienta para la medición de la productividad, con los propósitos de: "Cuantificar las actividades de los artesanos [de la construcción] identificar barreras a la productividad, implementar mejoras para disminuir 0 eliminar estas barreras y cuantificar el cambio en porcentajes de las actividades". Esta técnica ha sido validada por Goutt y colaboradores (Gouett, Haas, Goodrum y Caldas, 2011).

El análisis de actividad es un estudio transversal que se aplica en un proceso de mejora continua que consta de cinco pasos: la planificación del estudio comprende la definición de objetivos, población y la muestra y las categorías a ser consideradas; el segundo paso consiste en la recolección de datos en el campo; el tercero es el análisis de datos; el cuarto y quinto consisten en preparar un plan de mejoras y la implementación del mismo, que en el presente estudio no fueron desarrolladas, ya que sobrepasan los objetivos del trabajo .

El presente estudio fue concebido con un alcance descriptivo, ya que el trabajo observado fue clasificado en siete categorías, tal como han sido establecidas y definidas por el Cll. Estas son trabajo productivo en la realización de una actividad, trabajo preparatorio de la misma, el manejo de materiales, el manejo de 
herramientas y equipos, esperas ocasionadas por falta de materiales, equipo, instrucciones y otras; viajes y tiempo personal tomado en horas de trabajo.

La población de estudio seleccionada fueron las cuadrillas de carpintería de la empresa constructora Kosmox, trabajando en los encofrados en el segundo nivel del módulo D del Edificio de Ciencias de la Salud de la UNAH-VS. De estas, se seleccionó utilizar el método de muestreo de la cuadrilla, enfocándose en una de ellas, ya que de acuerdo con el CII "este enfoque provee información más detallada y permite a los observadores familiarizarse con los trabajadores de un oficio individuales".

Puesto que la cuadrilla se encontraba conformada por dos carpinteros y dos ayudantes, con el fin de garantizar la validez estadística y permitir hacer inferencias, se calculó que debían hacerse un mínimo de 45 observaciones al azar por hora, teniendo en consideraciones un error permisible del $5 \%$, un nivel de confianza del $95 \%$ y una proporción de ocurrencias del 50 \% (Oglesby, Parker y Howell, 1989; Serpell, 2002; Mora Aguirre, 2009; Construction Industry Institute, 2010; Gouett, Haas, Goodrum y Caldas, 2011).

Los datos fueron recolectados a partir de un video realizado en enero de 2013, para posteriormente registrarlos en hojas de trabajo preparadas para tal efecto (ver anexos). Se grabaron y analizaron dos horas que comprendieron el ciclo completo de la actividad de encofrado. Las observaciones se realizaron completamente al azar, para garantizar la posibilidad de que todos tuvieran igual oportunidad de ser observados. El uso de videos como medio de registrar operaciones de construcción con el fin hacer diagnósticos y mejoras de productividad, ha sido documentado por diferentes autores (Oglesby, Parker y Howell, 1989; Serpell, 2002; Mora Aguirre, 2009; Gong y Caldas, 2010).

Posteriormente, la información fue vaciada en una hoja de cálculo Excel 2010 para ser analizada. Las observaciones abarcan un ciclo completo de la actividad de encofrado de las columnas, con una duración aproximada de dos horas, entre las 10:00 a.m. y 11:00 a.m. Se totalizaron el número de observaciones para cada una de las siete categorías indicadas para el ciclo y se obtuvieron los porcentajes de tiempos dedicados por la cuadrilla, durante ese período, a cada categoría.

También se calcularon los porcentajes de tiempo dedicado por la cuadrilla a cada categoría, cada hora, para analizar si hay diferencias en el patrón conforme se consume el tiempo de trabajo. Adicionalmente, se estimó el tiempo que cada uno de 
los integrantes de la cuadrilla dedicó a las actividades categorizadas, para analizar si la cuadrilla analizada estaba conformada de manera equilibrada.

Gráfico 1. Tiempo dedicado a las diferentes actividades

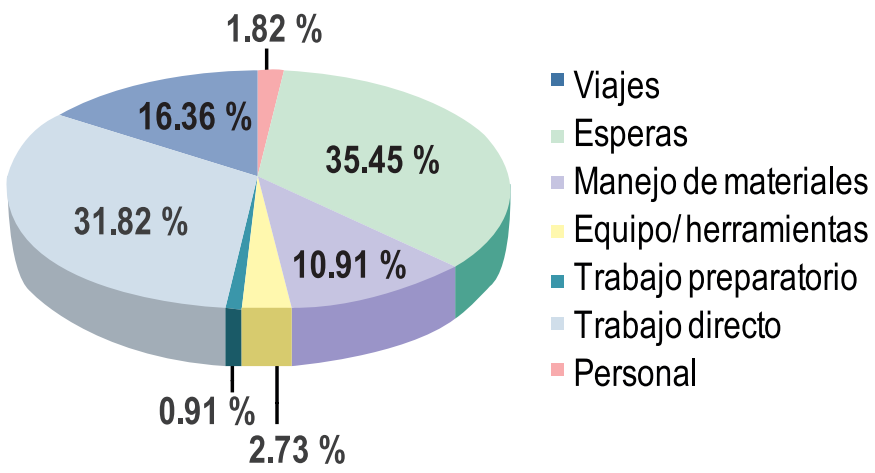

\section{RESULTADOS}

Los primeros resultados muestran que se obtuvieron un total de 110 observaciones recolectadas de la cuadrilla completa; de estas, 35 pueden catalogarse como trabajo productivo; 1 como trabajo preparatorio, siendo la categoría con menos observaciones; 3 correspondían al manejo de herramientas y equipo; se tuvieron 12 observaciones en referencia al manejo de materiales; la actividad con mayor cantidad de observaciones fue la de esperas: viajes tuvo 2 observaciones y se hicieron 18 observaciones que concernían a tiempo personal. En el gráfico 1 se muestran las proporciones obtenidas por cada uno.

Las observaciones de la cuadrilla completa, al ser cuantificadas por hora y analizadas, se muestran en el gráfico 2, en donde se aprecia que hay una disminución de un poco más del $4 \%$ en el trabajo productivo. El trabajo preparatorio no se presenta hasta después de las 11:00, mientras que el manejo de herramientas y equipo y los viajes desaparecen en ese mismo período de tiempo. 
Gráfico 2. Proporción del tiempo a las diferentes actividades

- Trabajo directo - Trabajo preparatorio Herramienta y equipo

-Esperas $\quad$ Viajes $\quad$-Tiempo personal

- Manejo de materiales

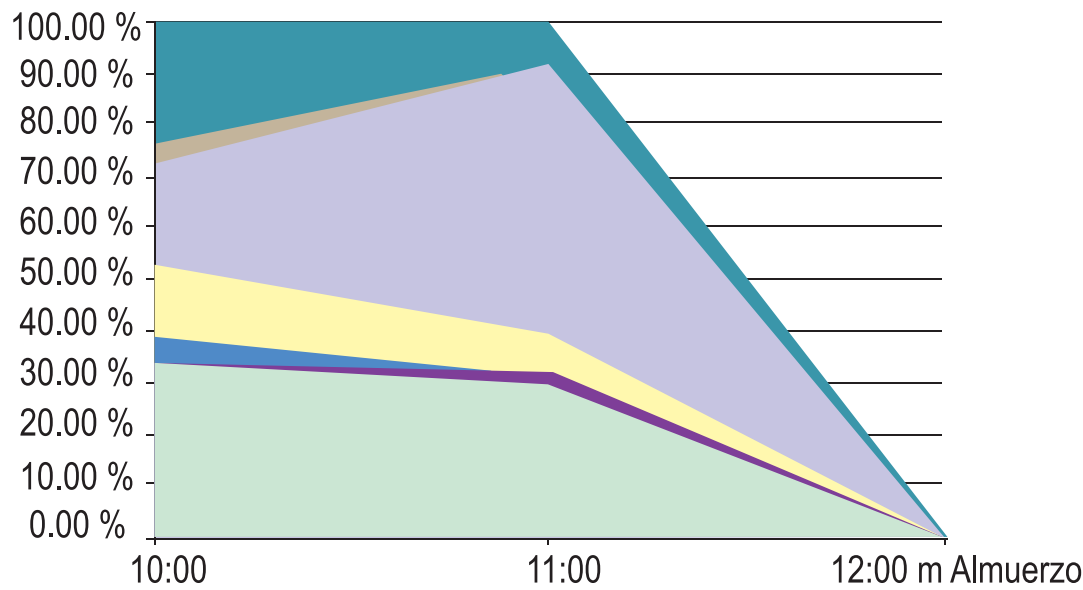

Existe una disminución del $6 \%$ en el manejo de materiales entre la primera y segunda hora. Igualmente, existe un incremento de más del $32 \%$ en el tiempo de espera en el mismo período y una disminución de casi $16 \%$ en la categoría de tiempo personal.

Al ordenar los datos por hora y después por cada miembro de la cuadrilla, se pudo encontrar que entre las 10:00 a.m. y las 10:00 a.m., el carpintero 1 dedicó un 33\% de su tiempo al trabajo productivo, un $20 \%$ del tiempo al manejo de herramientas, $13 \%$ de su tiempo al manejo de materiales y $20 \%$ a actividades personales. En el mismo período de tiempo, el carpintero 2 empleó el $56 \%$ de su tiempo en actividades productivas y un $6 \%$ a viajes. El ayudante 1 fue el que destinó menos tiempo al trabajo productivo y un $60 \%$ a actividades personales. El ayudante 2 empleó el $31 \%$ de su tiempo al manejo de materiales y un $15 \%$ a actividades personales. Todos pasaron entre un $7 \%$ y $38 \%$ esperando algún evento.

Entre las 11:00 a.m. y 12:00 m., todos los miembros dedicaron entre el 15\% y $36 \%$ a actividades de trabajo directo. El ayudante 1 realizó actividades de manejo de materiales en un $31 \%$ de su tiempo. Todos dedicaron entre el $43 \%$ y $69 \%$ de su tiempo inactivos o esperando a que algo sucediera. El carpintero 1 usó un $8 \%$ de su tiempo en actividades personales y el ayudante 2 empleó el $21 \%$ en similares actividades. En este lapso no hubo trabajo preparatorio, manejo de herramientas 0 de materiales por parte de ninguno de los integrantes de la cuadrilla. 


\section{DISCUSIÓN}

En esta sección se comparan y discuten los resultados obtenidos con resultados encontrados en la literatura internacional, ya que en el país la industria de la construcción carece de datos que sirvan de línea base. Se inicia comparando el trabajo productivo de la cuadrilla completa, que según Oglesby y colaboradores, se indica que para diferentes oficios el promedio del trabajo productivo es de un $36 \%$. Los mismos autores presentan resultados por oficio, si se toma en consideración que la cuadrilla está conformada por dos carpinteros y dos ayudantes; el porcentaje dedicado por la cuadrilla al trabajo productivo resulta ser del $36.5 \%$. De ambas maneras, al compararlo con el casi el $32 \%$ del estudio, se percibe que existe algún campo para hacer mejoras (ver gráfico 3 ).

Gráfico 3. Porcentaje de actividades ordenadas por hora y por miembro de la cuadrilla

\section{-Trabajo directo - Trabajo preparatorio $₫$ Equipo/ herramientas -Manejo de materiales $\|$ Esperas - Viajes Personal}

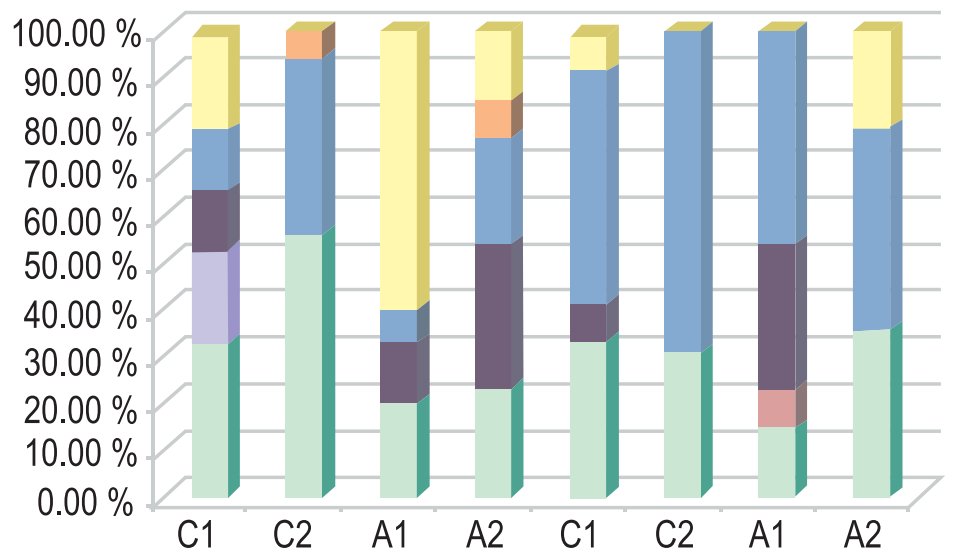

Hora: $10: 00$ a 11:00

Hora: $10: 00$ a $11: 00$

$$
\begin{array}{ll}
\mathrm{C} 1=\text { Carpintero } 1 & \mathrm{~A} 1=\text { Ayudante } 1 \\
\mathrm{C} 2=\text { Carpintero } 2 & \mathrm{~A} 2=\text { Ayudante } 2
\end{array}
$$

Lo anterior es consistente con los dato aportados por Serpell, quien establece que el promedio nacional de Chile era del $38 \%$, variando entre el $31 \%$ en construcciones de edificios en extensión, a un $47 \%$ en edificios en elevación. En el caso de la UNAH-VS, se debe considerar que el complejo de Ciencias de la Salud es un punto intermedio, tiene edificios de más de tres pisos, pero también puede considerarse 
una construcción en extensión, por lo que puede estimarse el trabajo productivo en un $36 \%$ como promedio de ambos extremos, lo que coincide con lo manifestado anteriormente. Al notar que el trabajo directo es menor al $32 \%$, se hace evidente que existe un pequeño margen para hacer mejoras.

Los resultados presentados por los autores antes citados fueron desarrollados utilizando la técnica del muestreo del trabajo, quienes establecían solo tres categorías de análisis: trabajo productivo, contributorio y no contributorio. El problema se presenta al tratar de comparar las otras seis categorías que establece la técnica del análisis de actividad. En tal sentido, a fin de poder compararlos con los resultados de los autores antes mencionados, se tendrían que hacer las siguientes transformaciones: el trabajo contributorio definido por Oglesby y por Serpell, equivale someramente a las categorías trabajo preparatorio, manejo de materiales y manejo de herramientas. La definición del trabajo no contributorio propuesta por los dos autores antes citados, corresponde a grandes rasgos al tiempo de esperas, viajes y personal.

El trabajo contributorio definido por Oglesby lo establece en un $31 \%$ y por Serpell, que lo establece en un $36 \%$, equivale de alguna manera a las categorías trabajo preparatorio, manejo de materiales y manejo de herramientas, que sumando sus respectivos resultados equivale a un $14.55 \%$; el cual es bastante bajo. Este resultado es consistente con lo señalado por el Cll, ya que las proporciones correspondientes a estas categorías se realizan a primera hora de la mañana, disminuyendo a finales de la mañana cuando las cuadrillas están por tomar el receso del mediodía para almorzar. Dado que el estudio fue realizado entre 10:00 a.m. y 12:00 m., los resultados son consistentes con los presentados por el Cll.

Comparando resultados obtenidos con la técnica del análisis de actividad en el presente trabajo, con los resultados de Gouett en seis proyectos en los Estados Unidos, podemos observar que los autores encontraron que el promedio dedicado al trabajo preparatorio en estos seis proyectos fue del $12.1 \%$. En cambio, el porcentaje de tiempo dedicado a esta actividad en el edificio de Ciencias de la Salud fue del $0.91 \%$, el cual está muy debajo por el de Gouett. Una explicación a esta discrepancia podría encontrarse en el trabajo del Cll, el que señala que esta categoría incluye actividades que no pueden ni deben ser eliminadas, como charlas de seguridad o instrucciones del trabajo a realizar, que se realizan a primera hora del trabajo, considerando que este estudio fue desarrollado al final de la mañana.

De similar manera, el tiempo dedicado al manejo de equipo y herramientas $(2.73 \%)$ en este proyecto resultó menor que el promedio encontrado por Gouett (11.4 \%). 
También este resultado puede atribuirse a la misma razón enunciada anteriormente, es decir, la hora en que se realizó el estudio, ya que si existen problemas con las herramientas se resuelven a primera hora, no al momento del estudio. El tiempo dedicado al manejo de materiales en este estudio (10.91\%) resultó ser el doble que el reportado por Gouett (2011), quien señala como posibles causas de este tipo de problema a la pobre planificación del proceso constructivo y a una cuadrilla de trabajo mal balanceada.

El trabajo no contributorio, según las definiciones utilizadas por Oglesby (1989), se establece en un $31 \%$ y por Serpell en un $26 \%$, comparado con el $53 \%$ obtenido lo que indica que existe un problema. Al compararlo con los resultados obtenidos por Gouett y colaboradores, se encontró que el promedio en seis proyectos, el tiempo dedicado a las esperas fue menor al $15 \%$; en cambio, en el proyecto analizado fue mayor del $35 \%$. En ese mismo trabajo, los autores atribuyen como probables causas de las esperas a una pobre planeación, una cuadrilla desbalanceada 0 a esperas de instrucciones, equipo, herramientas o materiales. Por lo observado en el campo, podría descartarse la última causa. En el planteamiento de acciones de mejoras, si el alcance del trabajo lo hubiese permitido, tendrían que estudiarse a mayor profundidad las dos primeras causas.

El porcentaje de tiempo dedicado a viajes reportado por Gouett (2011), fue cerca del $15 \%$; pero, en el presente trabajo fue del $1.82 \%$. Nuevamente, los resultados presentados por el Cll (2010) indican que esta actividad disminuye al final de la mañana. En cuanto a la categoría que describe la proporción de tiempo dedicado por la cuadrilla a actividades personales, en el trabajo de Gouett se obtuvo un promedio del $7.7 \%$, mientras que en el presente trabajo se observó que más del 16 $\%$ de tiempo lo dedicaron a estas actividades. De acuerdo a Goutt, las causas más frecuentes son inicios tardíos y finales tempranos, pobre presencia de la administración del proyecto y largas horas de trabajo.

Al analizar el gráfico 2, se puede observar cómo la proporción del tiempo dedicado a cada actividad varía en el transcurso del día, lo que coincide con lo enunciado por el CII (2010). Así, la porción dedicada al trabajo productivo se reduce a medida que se acerca la hora del almuerzo, consistente con lo manifestado por Serpell, la producción diaria disminuye alrededor del mediodía. Al respecto, es notorio el aumento en el tiempo de esperas en la última hora analizada, antes del almuerzo; igualmente lo es la disminución de tiempo personal en este período de tiempo. Estos dos resultados no son consistentes con los del trabajo reportado por el CII (2010), por eso es un buen indicador de que el método empleado tiene problemas. Sin embargo, una de las ventajas de realizar el trabajo a partir de un video, es que 
permite realizar investigaciones posteriores para encontrar una explicación a estos fenómenos.

La respuesta puede estar en los resultados presentados en el gráfico 3 , que muestra las proporciones de tiempo dedicado a cada actividad por cada miembro de la cuadrilla, pues al hacerlo de esta manera, permite observar la cantidad de tiempo dedicado por cada miembro a cada actividad, similar, en cierta manera, a una carta de balance de cuadrilla (Oglesby, Parker y Howell, 1989). En este aspecto, lo primero que se puede notar es que durante la primera hora solo los carpinteros se dedican al trabajo productivo en un porcentaje razonable de su tiempo, pero existe grandes porciones de tiempo dedicado a esperas y tiempo personal.

Luego, en la segunda hora, decae el porcentaje dedicado al trabajo productivo y el tiempo dedicado a actividades personales, aumentando considerablemente el tiempo dedicado a esperar. Los resultados representados en el gráfico 3 son similares al de las cartas de balance de cuadrilla, que aplica lo señalado por Oglesby (1989), puesto que este gráfico: "No necesariamente demuestra la efectividad o la eficiencia de una operación, ya que mostrarse ocupado no es sinónimo de utilizar un buen método". Lo que indica es que el método de transformación seguido en este proceso, tiene deficiencias sin cambiar la manera cómo se ejecutaba este trabajo, de modo que la conformación de la cuadrilla debe de ser revisada, probablemente quitando uno de los ayudantes de esta cuadrilla, pero las mejoras a esta operación pueden ser más significativas, lo cual se sale del propósito del presente trabajo.

\section{LIMITACIONES}

El presente trabajo de investigación fue realizado basado en videos grabados por un grupo de estudiantes de la MICGP, como parte del trabajo asignado en una asignatura, pero no se contó con el tiempo para realizar un trabajo que abarcara varios días, lo que hubiera facilitado obtener información que muestre la variación en el transcurso del día; asimismo, se limitó al trabajo solo de las operaciones de encofrado, en lugar de considerar un aspecto más amplio y, más importante aún, hubo restricciones para diagnosticar el problema, en lugar de aplicar un proceso continuo de mejora.

\section{CONCLUSIONES}

Parafraseando las palabras de Lord Kelvin: "Si se puede medir, se puede mejorar", en este trabajo se logró medir y describir a qué actividades y en qué proporción se 
dedica el tiempo de trabajo en una operación de encofrado de columnas en el Edificio de Ciencias de la Salud. Al analizar esta distribución del tiempo dedicado a actividades productivas, esperas y actividades personales, se pudo observar que existen indicios para espacios de mejoras en el proceso productivo.

Cuando se estudió la información mostrada en el gráfico 2, se pudo determinar que existen variaciones en cuanto a la forma en que la cuadrilla distribuye su tiempo en el transcurso del día, lo que es consistente con la literatura analizada, pudiendo observarse que la productividad baja cerca del mediodía, tal como lo explica Serpell (2002). El tiempo dedicado a esperas y actividades personales sugiere que existen problemas en el proceso productivo, encontrando en la literatura consultada que algunas causas a investigar son la pobre planificación, cuadrillas con exceso de personal y mejor administración del plantel del proyecto (Construction Industry Institute, 2010).

El analizar la manera en la que cada miembro de la cuadrilla ha distribuido su tiempo entre las diferentes categorías de estudio, permite observar el aporte que cada uno de ellos está haciendo al producto final, indicando ineficiencias del método utilizado en el proceso. Se puede apreciar como el trabajo productivo baja en la última hora del proceso, siendo extremadamente bajo en el ayudante 1. Adicionalmente, se puede apreciar la cantidad excesiva dedicada a esperas, las que aumentan considerablemente en la última hora para toda la cuadrilla, igualmente el tiempo dedicado a actividades personales por el carpintero 1 y los dos ayudantes.

Finalmente, al observar y comparar los resultados obtenidos por la técnica del análisis de actividades con los del muestreo del trabajo, se pudo comprender que debido a la mayor cantidad de observaciones requeridas y al mayor número de categorías utilizadas por la primera, los resultados que esta técnica proporciona permite hacer evaluaciones descriptivas que indican de manera más detallada adonde deben orientarse los siguientes esfuerzos de mejora.

\section{AGRADECIMIENTO}

A la Secretaría Ejecutiva de Administración de Proyectos de Infraestructura (SEAPIUNAH), a los representantes de Constructora Kosmox y del Gabinete Técnico y a todos los trabajadores de la construcción que participaron en este proyecto, por la colaboración prestada. 


\section{BIBLIOGRAFÍA}

Alarcón C., L. F. (2012). Herramientas para identificar y reducir pérdidas en proyectos de construccíon. Revista Ingeniería de Construcción, 15, 37-45.

Business Roundtable. (1983). More construction more money: summary report of the Construction Industry Cost Effectiveness Project. New York.

Construction Industry Institute. (July, 2010). Guide to Activity Analysis. University of Texas. Craft Productivity, Program Research Team, Austin.

Court, P. F.; Pasquire, C.\& Gibb, A. (2009). A lean and agile construction system as a set of countermeasures to improve health, safety and productivity in mechanical and electrical construction. Lean Construction Journal, 61-76.

Diario La Prensa. Sección Economía. (13 de octubre de 2011). Baja ejecución margina a empresas constructoras. Diario La Prensa, 84.

Gong, J. \& Caldas, C. H. (Mayo/junio de 2010). Computer Vision-Based Video Interpretation Model for Automated Productivity Analysis of Construction Operations. Journal of Computing in Civil Engineering, 252-263.

Gouett, M. C.; Haas, F. ; C.T., Goodrum, P.M. \& Caldas, C.H. (2011). Activity analysis for direct-work rate improvement in construction. Journal of Construction Engineering and Management, 1118-1124.

Jenkins, J. L. \& Orth, D.L. (2003). Productivity Improvement through Work Sampling. AACE International Transactions, CSC.05.

Martínez C., L. F. \& Alarcón C., L.F. (Julio - diciembre de 1988). Programas de mejoramiento de la productividad para obras de construcción. Revista do Ingeniería de Construcción,(5).

Martínez C., L.F.; Verbal R.R. \& Serpell B.A. (Enero-junio de 1990). Recomendaciones para aumentar la productividad en la construcción. Revista Ingeniería de Construcción, 1-11

Mora Aguirre, M. A. (2009). Utilización de imágenes digitales para el mejoramiento de la productividad de operaciones de construcción. Tesis. Universidad de Chile, Facultad de Cienicas Físicas y Matemáticas, Ingeniería Civil.

Oglesby, C.H., Parker, H. W. \& Howell, G. A. (1989). Productivity Improvement in Construction. New York: McGraw Hill.

Orth, D.L. \& Jenkins, J.L. (2004). Mechanical and General Construction Productivity Results. Cost engineering, 46 (3), 33-36.

Proceso Digital. (5 de noviembre de 2012). Coalianza beneficia a empresas extranjeras denuncian empresarios de la construcción. Disponible en: http://www.proceso.hn/2012/11/05/Econom\%C3\%ADa/Coalianza.beneficia.a/5 9736.html

Ramírez, R.R.; Alarcón, L.F. \& Knights, P. (Julio de 2004). Benchmarking System for Evaluating Management Practices in the Construction Industry. Journal of Management in Engineering, 20 (3), 110-117. 
Saxon, D. (17 de diciembre de 2007). In praise of Lord Kelvin. Recuperado el 5 de agosto de 2013. Disponible en

http://physicsworld.com/cws/article/indepth/2007/dec/17/in-praise-of-lord-kelvin

Serpell B., A. (2002). Administracion de operaciones de construcción. México: Alfaomega.

Vásquez, K. (5 de noviembre de 2012). Construcción en Honduras tendrá un 2013 "negro". Diario el Heraldo.

\section{ANEXO}

\begin{tabular}{|c|c|c|c|c|c|c|}
\hline \multicolumn{7}{|c|}{ Fecha } \\
\hline \multicolumn{2}{|c|}{ Observación \# } & & T. Inicio & $10: 00$ & T. Final & $11: 00$ \\
\hline \multirow{11}{*}{$\begin{array}{l}\text { 옹 } \\
\text { 홍 } \\
\text { 융 }\end{array}$} & Categoría & & & Oficios & & \\
\hline & Conteo & & 2 & & & \\
\hline & Oficio & Car & tero & & ante & \\
\hline & Identificador & & & & & \\
\hline & $\begin{array}{l}\text { Trabajo } \\
\text { directo }\end{array}$ & 5 & 9 & 3 & 3 & 20 \\
\hline & $\begin{array}{l}\text { Trabajo } \\
\text { preparatorio }\end{array}$ & 0 & 0 & 0 & 0 & 0 \\
\hline & $\begin{array}{l}\text { Herramienta } \\
\text { / Equipo }\end{array}$ & 3 & 0 & 0 & 0 & 3 \\
\hline & $\begin{array}{l}\text { Manejo de } \\
\text { materiales }\end{array}$ & 2 & 0 & 2 & 4 & 8 \\
\hline & Esperas & 2 & 6 & 1 & 3 & 12 \\
\hline & Viajes & 0 & 1 & 0 & 1 & 2 \\
\hline & Personales & 3 & 0 & 9 & 2 & 14 \\
\hline
\end{tabular}

